\title{
Lentivirus vector-mediated gene transduction of CNGRC peptide in rat adipose stem cells
}

\author{
DU MENG ${ }^{*}$, RUI LIU*, LI PEI, LEI HOU, QIAN NING, QING YU, LU FENG and XINHAN ZHAO \\ Department of Oncology, First Affiliated Hospital of Medical School of Xi'an Jiaotong University, \\ Xi'an, Shaanxi 710061, P.R. China
}

Received February 6, 2014; Accepted November 7, 2014

DOI: $10.3892 / \mathrm{mmr} .2014 .3043$

\begin{abstract}
The aim of the present study was to investigate the feasibility of lentiviral-mediated Cys-Asn-Gly-Arg-Cys (CNGRC) peptide gene transduction in adipose stem cells. Adipose stem cells were prepared using enzymatic digestion and repeated adherence methods and identified in culture by immunofluorescence staining of surface markers. The pluripotency of the cultured adipose stem cells was confirmed by their induced differentiation into bone and fat cells. Following polymerase chain reaction amplification, the gene sequence for the CNGRC peptide was cloned into a lentiviral vector, which was then co-transfected into $293 \mathrm{~T}$ cells with packaging plasmids Helper 1.0 and Helper 2.0. The lentiviruses carrying the CNGRC peptide gene were then harvested and used to transfect adipose stem cells. Following transduction, expression of CNGRC in adipose stem cells was detected using western blot analysis. Adipose stem cells in culture were successfully induced to differentiate into adipocytes and osteoblasts and the lentiviral vector containing CNGRC-3Flag-EGFP was successfully constructed. Following transduction, western blot analysis and immunofluorescence staining demonstrated expression of the CNGRC protein in adipose stem cells. This suggested that adipose stem cell lines expressing the CNGRC peptide were successfully established.
\end{abstract}

\section{Introduction}

Vascular niches of cancer stem cells formed by microvessels in a tumor have been considered a cause of local tumor recurrence and metastasis $(1,2)$. Therefore, microvessels in

Correspondence to: Professor Xinhan Zhao, Department of Oncology, First Affiliated Hospital of Medical School of Xi'an Jiaotong University, 277 Yanta West Road, Xi'an, Shaanxi 710061, P.R. China

E-mail: zhaoxinhanprof@163.com

*Contributed equally

Key words: adipose stem cells, cell surface markers, green fluorescence protein, lentivirus vector, CNGRC peptide stem cell niches constitute a new target for cancer therapy and destruction of certain clusters of microvessels may assist in eliminating cancer stem cells. Since stem cells can be recruited to stem cell niches, a process termed stem cell homing, the use of stem cells as vectors for gene therapy has become an important topic of discussion in previous years (3).

Adipose stem cells (ADSCs) isolated from adipose tissue have previously been identified in adults (4) and have phenotypes and differentiation potentials similar to those of bone marrow mesenchymal stem cells (5). Numerous studies have demonstrated that ADSCs possess an active homing capacity for multiple types of cancer, including ovarian cancer, glioma and metastatic lung cancer (4,6-8). Additionally, ADSCs are easily isolated, cultured and transfected in vitro and can survive following transplantation into animals (3). Therefore, ADSCs are good candidates for transporting anti-angiogenesis drugs in gene therapy as they can migrate to the microvessels of cancer stem cell niches, destroy the microvessels and ultimately eliminate the cancer stem cells.

In a previous study, a DNA sequence expressing Asn-Gly-Arg (NGR) and the cyclic peptide Cys-Asn-Gly-Arg-Cys (CNGRC) was designed that could specifically penetrate breast cancer cells (9). Additionally, in vitro experiments have indicated that the CNGRC peptide has antitumor activity (9). NGR is a tri-peptide motif that can specifically bind to vascular endothelial cells of newly generated blood vessels and such binding is mediated by amino peptidase N (CD13). CD13 is a transmembrane protein with a molecular weight of $140,000 \mathrm{Da}$. It is mainly expressed in solid tumors and the blood vessels of new tumors, but is rarely expressed in normal blood vessels. NGR peptide not only binds to new vessels through CD13, but can also undergo deamination of asparagine to form the isomer, isoDGR. isoDGR can regulate adhesion and proliferation of epithelial cells and has a higher affinity than NGR for blood vessels in new tumors (10). Following binding, NGR peptide can be internalized into vascular endothelial cells by receptor-mediated endocytosis. Based on this principle, NGR peptide can carry compounds and particles, including cytotoxic drugs, cytokines, virus particles and fluorescent compounds to new vessels, which can markedly improve the efficacy of targeted therapy (11). A number of studies have used NGR motif containing peptides for ligand-mediated imaging and treatment of tumors with new blood vessels (12-15). For example, Corti (16) et al used doxorubicin (DOX)-NGR conjugates to treat human metastatic 
tumors in nude mice. Compared with free DOX, DOX-peptide conjugates not only improved the efficacy of breast cancer treatment, but also significantly reduced toxicity to the liver and heart. Curnis et al (17) demonstrated that use of tumor necrosis factor-NGR conjugates improved drug permeability and the therapeutic effects of tumor necrosis factor by 8-10-fold. However, stem cells expressing NGR peptide and its conjugates were not available for assessment.

To establish an NGR expressing stem cell line, it is essential to select a gene delivery vector that can stably express CNGRC peptide for a long period of time. Eukaryotic viral vectors, including adenovirus vectors, adeno-associated vectors, retrovirus vectors and lentiviral vectors have high transduction efficiencies and evident targeting capacities and are most commonly used in gene therapy. However, retroviral vectors can only infect dividing cells and cannot accommodate DNA fragments $>8 \mathrm{~kb}$ (18). Additionally, adenovirus vectors cannot achieve long-term expression of exogenous genes and repeated application of the adenovirus can lead to immune responses (19). By contrast, the human immunodeficiency virus derived lentiviral vector can infect dividing cells and non-dividing cells and produce long-term stable expression of exogenous genes, without inducing an immune response (20). Compared with other conventional transduction methods, including liposome transduction, calcium phosphate transduction and electroporation, use of a lentivirus can also efficiently infect cells that are difficult to transfect, including nerve cells, myocardial cells, endothelial cells and cells grown in suspension $(21,22)$. Therefore, lentiviral vectors are the most satisfactory gene transfer vectors for use in gene therapy.

In the present study, an enzymatic digestion and repeat adherence methods were used to isolate highly purified ADSCs and the feasibility of using a lentiviral vector to express CNGRC peptide in ADSCs was investigated.

\section{Materials and methods}

Animal care and maintenance. Sprague Dawley (SD) rats aged 3 months and weighing 180-250 g were maintained at the Experimental Animal Center of Medical School of Xi'an Jiaotong University (Xi'an, China). All experimental manipulations were performed in accordance with general guidelines of the Association for Assessment and Accreditation of Laboratory Animal Care. The Animal Care and Use Committee of Xi'an Jiaotong University reviewed and approved all animal-related procedures.

Isolation and culture of primary ADSCs. Bilateral samples of inguinal subcutaneous adipose tissue $(400-600 \mathrm{ml})$ were collected from adult male SD rats. The samples were washed, cut into sections $\left(1^{3} \mathrm{~mm}\right)$ and digested in $0.1 \%$ collagenase I at $37^{\circ} \mathrm{C}$ on a shaker $(100 \mathrm{x} \mathrm{g})$ for $60 \mathrm{~min}$. Suspended fat was removed by centrifuging twice at $1,200 \times \mathrm{g}$ for $12 \mathrm{~min}$. The cells were then filtered and suspended in Dulbecco's modified Eagle's medium (DMEM)/F12 containing 10\% fetal bovine serum and penicillin/streptavidin and cultured for $72 \mathrm{~h}$ at $37^{\circ} \mathrm{C}$, in a $5 \% \mathrm{CO}_{2}$ humidified incubator (Thermo Fisher Scientific, Rockford, MA, USA). After 72 h, non-adherent red blood cells were removed by changing the culture medium. Following reaching $70-80 \%$ confluence, the fat cells were passaged at a ratio of 1:2 into two T-25 cell culture flasks. The cells were then cultured at $37^{\circ} \mathrm{C}$ in a $5 \% \mathrm{CO}_{2}$ humidified culture container. The culture medium was changed every 2-3 days.

Immunofluorescence staining. Surface markers CD29, CD34, CD106 and CD90 were used to identify ADSCs. Following reaching $80 \%$ confluence, at the third passage ADSCs were stained using fluorescence-labeled antibodies, phycoerythrin (PE)-conjugated mouse-anti-rat CD106 monoclonal antibody, PE mouse-anti-rat CD34 monoclonal antibody, PE mouse-anti-rat CD90 monoclonal antibody and PE mouse-anti-rat CD29 monoclonal antibody (1:5,000; BioLegend, San Diego, CA, USA). Cells were visualized and images were captured using an inverted fluorescence microscope (ECLIPSE Ti; Nikon, Tokyo, Japan).

Adipogenic induction. ADSCs at the third passage were induced by addition of an adipogenic inducer solution containing $10 \%$ fetal bovine serum, $1 \mu \mathrm{mol} / 1$ dexamethasone, $0.5 \mathrm{mmol} / 1$ 3 -isobutyl-1-methyl xanthine and $1 \mu \mathrm{mol} / 1$ indomethacin in DMEM/F12. Control cells were cultured in DMEM/F12 containing $10 \%$ fetal bovine serum. Cells were maintained at $37^{\circ} \mathrm{C}$ in a $5 \% \mathrm{CO}_{2}$ incubator and the medium was changed every 3 days. Following a 2 week period of induction, the cells were stained with Oil Red $\mathrm{O}$ and observed under an inverted microscope (TMS; Nikon).

Osteogenic induction. ADSCs at the third passage were induced by addition of induction media containing $1 \mu \mathrm{mol} / 1$ dexamethasone, $0.5 \mathrm{mmol} / 13$-isobutyl-1-methylxanthine and $1 \mu \mathrm{mol} / \mathrm{l}$ indole indomethacin. Control cells were cultured in DMEM/ F12 containing 10\% fetal bovine serum. Cells were maintained at $37^{\circ} \mathrm{C}$ in a $5 \% \mathrm{CO}_{2}$ incubator and the medium was changed every 3 days. Following a 3 -week induction period, the cells were stained with alizarin red and cells exhibiting calcium nodule formation were observed under an inverted microscope (TMS; Nikon).

Lentivirus packaging and quantitative fluorescence polymerase chain reaction (PCR). Aliqots of 293T cells (GeneChem, Montreal,Canada) were cultured in high-glucose DMEM supplemented with $10 \%$ fetal bovine serum. Recombinant virus plasmid Ubi-CNGRC-3Flag-EGFP and packaging plasmids (Helper 1.0 and Helper 2.0) were prepared using an EndoFree Plasmid Maxi kit (Qiagen, Amsterdam, The Netherlands). Three plasmids were co-transfected into $293 \mathrm{~T}$ cells using Lipofectamine 2000 (Life Technologies, Grand Island, NY, USA). After a $48 \mathrm{~h}$ transduction period, the supernatant containing lentiviral particles was harvested and concentrated by super-speed centrifugation at 4,000 x $\mathrm{g}$ for $10 \mathrm{~min}$. Viral titers were measured by quantitative PCR, using GAPDH as an internal control. The $\mathrm{Ct}$ value was defined as the number of cycles when the fluorescent signal reached a specified threshold. The sequences of primers were as follows: EGFP, forward 5'-TGCTTCAGCCGCTACCC-3' and reverse 5'-AGTTCACCTTGATGCCGTTC-3'; GAPDH, forward 5'-TGACTTCAACAGCGACACCCA-3' and reverse 5'-CACCCTGTTGCTGTAGCCAAA-3'.

Lentiviral infection of ADSCs. Concentrated lentiviral solutions of LV-EGFP and LV-CNGRC-3Flag-EGFP were added 

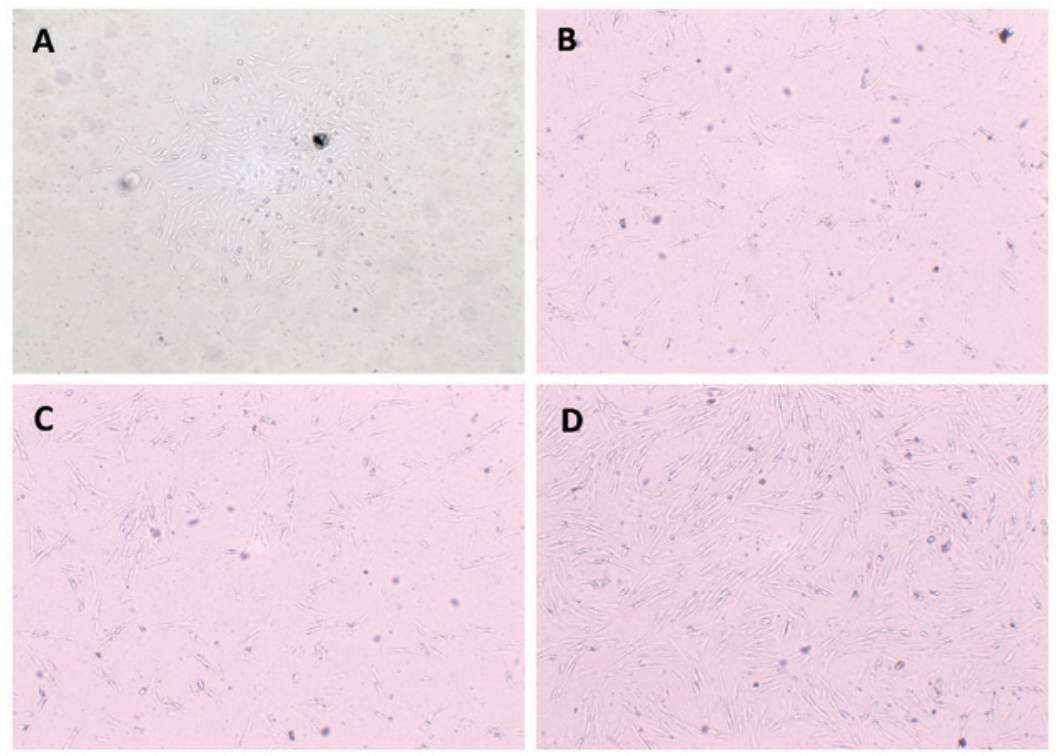

Figure 1. ADSCs at different passages observed under an inverted microscope. (A) Primary ADSCs cultured for 3 days (magnification, x600). (B) ADSCs at passage 1 cultured for 11 days (magnification, x600). (C) ADSCs at passage 3 cultured for 3 days (magnification, x400). (D) ADSCs at passage 4 cultured for 3 days (magnification, $\mathrm{x} 400$ ). ADSCs, adipose stem cells.

into two wells of cultured ADSCs, respectively, once the cells reached $40 \%$ confluence. Enhanced infection solution was then added to reach a total incubation volume of $2 \mathrm{ml}$. After $12 \mathrm{~h}$ of incubation, the cell culture medium was changed and $72 \mathrm{~h}$ later, images of the cells expressing EGFP were captured under an inverted fluorescence microscope (ECLIPSE Ti; Nikon). Images were processed using NIS-Elements imaging software (Nikon Instruments, Inc., Tokyo, Japan).

Immunoblot analysis. Stable transfected ADSCs were selected with puromycin (Sigma-Aldrich, St. Louis, MO, USA) and CNGRC expression levels of cells at the third passage (12 days after transduction) were identified by western blot analysis. Total protein was extracted from ADSCs transfected with LV-EGFP and LV-CNGRC-3Flag-EGFP, respectively. Following denaturation, the protein samples were centrifuged at $12,000 \mathrm{x} g$ for $2 \mathrm{~min}$. The target proteins were hybridized with mouse anti-flag tag monoclonal antibody (1:1,000; Proteintech, Chicago, IL, USA), mouse anti- $\beta$-actin monoclonal antibody (1:5,000; Proteintech) and horseradish-peroxidase-conjugated goat anti-mouse IgG polyclonal antibody (1:10,000; CWBio, Beijing, China) IgG. Enhanced chemiluminescent substrates (Pierce Biotechnology, Inc., Rockford, IL, USA) were used to detect the signals of targeted proteins.

Statistical analysis. All statistical analyses were performed using SPSS 16.0 software (SPSS, Inc., Chicago, IL, USA). Data are expressed as the mean \pm standard error of the mean. The paired-sample t-test was used for comparisons among multiple groups and $\mathrm{P}<0.05$ was considered to indicate a statistically significant difference.

\section{Results}

Successful isolation and culture of ADSCs. ADSCs were isolated from rat adipose tissue by use of enzymatic diges- tion. Twenty-four hours after seeding, several transparent adherent cells with round or oval morphology were visible under a microscope and at 4 days after seeding, additional ADSCs with fusiform and polygonal morphology were observed. At 10-11 days after seeding, the cell clones had significantly expanded, become connected to each other and reached $80-90 \%$ confluence. Cells with long spindle-shaped morphology were closely arranged in a swirling pattern with multilayer cells in the center. Following passage, the ADSCs demonstrated a higher growth rate. Without alterations in cell morphology, the doubling time of these cells was $70 \mathrm{~h}$ and the cells could be successfully sub-cultured (Fig. 1).

ADSCs express typical surface markers in culture. ADSCs were identified by cell surface markers (23). Previous studies reported that ADSCs expressed the cell surface markers CD90, CD29, CD49e, CD54, CD55, CD63, CD73 and CD105, but did not express CD11b, CD34, CD31, HLA-DR and CD117 (24,25). Zuk et al (26) revealed that ADSCs expressed CD49d but not CD106, while bone marrow mesenchymal stem cells expressed CD106 but not CD49d. In the present study, $>80 \%$ of cultured cells were CD29 and CD90 double positive (Fig. 2C and D) and CD106 and CD34 double negative (Fig. 2A and B). These findings indicated that ADSCs were successfully isolated and cultured in vitro.

ADSCs differentiate to form adipocytes in culture. Adipogenic inducers were added to cultures of ADSCs to determine their capacity for adipogenesis. Following induction for $24 \mathrm{~h}$, the cells became smaller in size and the cytoplasm began to retract and demonstrated a small square morphology. The refractive index of these cells was also enhanced. At 3-4 days after induction, cell proliferation slowed significantly and small, round, shiny lipid droplets had formed in the cytoplasm. Observations made at longer time periods following induction revealed enlargement of 

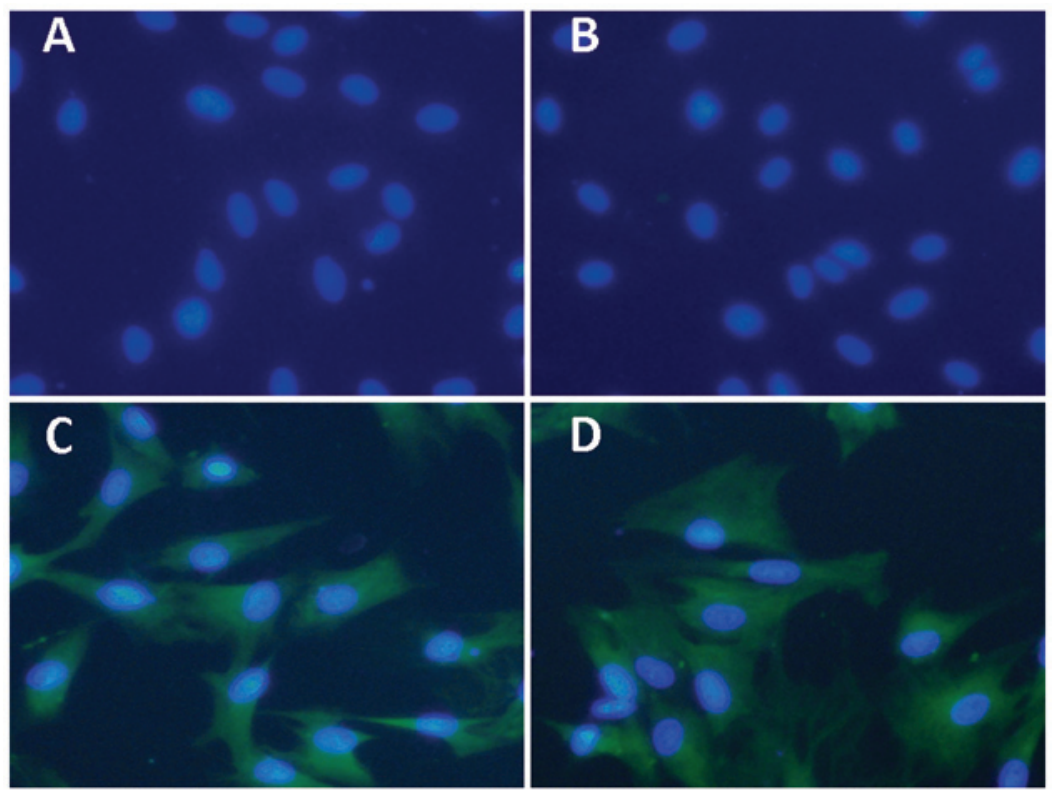

Figure 2. Surface markers expressed in ADSCs. Cultured ADSCs did not express (A) CD34 and (B) CD106, but did express (C) CD29 and (D) CD90. Green: surface markers. Blue: 4',6-diamidino-2-phenylindole (magnification, x2,000). ADSCs, adipose stem cells.
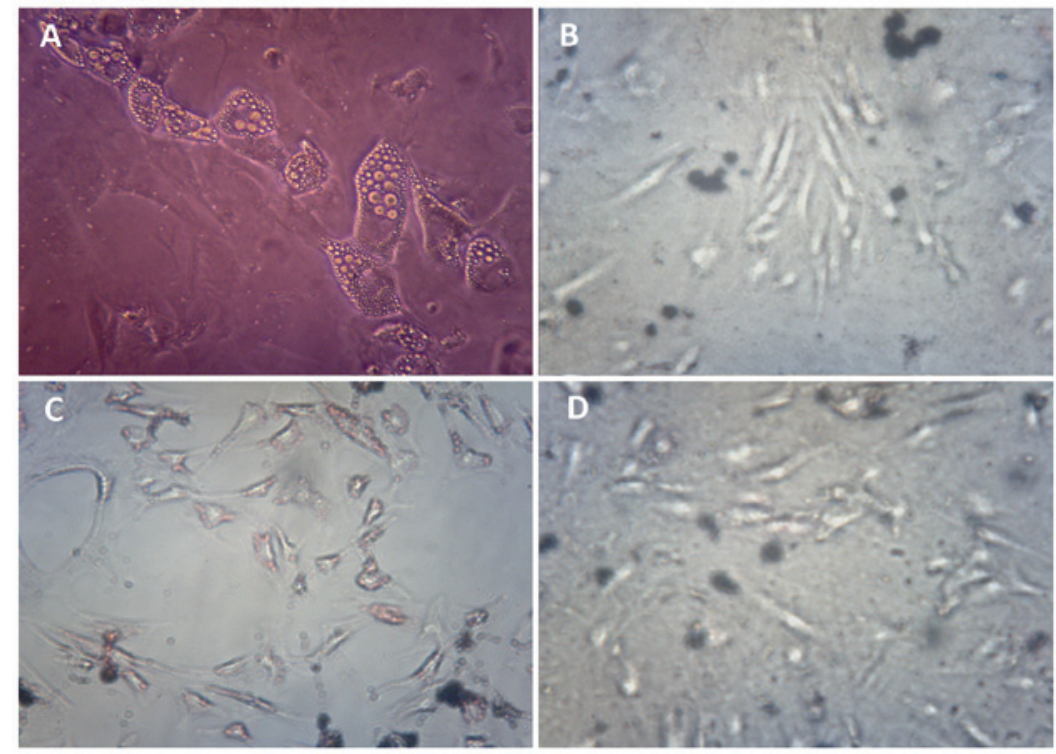

Figure 3. ADSCs following adipogenic induction. (A and B) ADSCs were cultured in induction medium (A, magnification, x2,000) or normal medium (B, magnification, x600) for 1 week. (C and D) Oil Red O staining-labeled fat droplets in ADSCs cultured in induction medium for 2 weeks (C, magnification, $\mathrm{x} 600$ ) but not in cells cultured in normal medium (D, magnification, x600). ADSCs, adipose stem cells.

lipid droplets in the cytoplasm and increased cell numbers (Fig. 3A and C). At 2 weeks after induction, ADSCs were stained with Oil Red O, which revealed the presence of various sized round red particles and fat droplets in the cytoplasm. By contrast, no significant difference was identified in cell size or formation of small lipid droplets in cells not treated with the adipogenic inducer (Fig. 3B and D).

ADSCs differentiate to form osteocytes in culture. Osteogenic inducers were added to cultures of ADSCs to examine their capacity for osteogenesis. After 3 days of induction, the morphology of ADSCs altered from a long spindle shape into a round or irregular shape. Additionally, cell body size increased, cellular projections disappeared and the cell proliferation rate decreased. One week after induction, the cells formed colonies, the nuclei became rounded and larger and additional small black particles appeared in the cytoplasm. Following longer periods of induction, the cells became distributed surrounding the colonies and grew in an overlapping manner. At 3 weeks after induction, the cells had secreted large quantities of extracellular matrix and formed growing granular calcium nodules of varying sizes (Fig. 4A). The mineralized nodules were distributed in colonies and had a reddish-brown color following alizarin red staining, confirming calcium deposition (Fig. 4C). By contrast, there was no calcium deposition in cells that were not subjected to induction (Fig. 4B and D). 
Table I. Relative quantities of CNGRC mRNA in non-transfected, LV-EGFP-transfected and LV-CNGRC-3Flag-EGFP-transfected adipose stem cells.

\begin{tabular}{lccc}
\hline Relative mRNA level & LV $^{\mathrm{a}}$ & LV-EGFP $^{\mathrm{b}}$ & LV-CNGRC-3Flag-EGFP $^{\text {ab }}$ \\
\hline (Average \pm STDEV) & $1.054 \pm 0.413$ & $0.967 \pm 0.538$ & $1883.897 \pm 145.304$
\end{tabular}

$\mathrm{P}<0.05$, LV-CNGRC-EGFP vs. LV; P<0.05, LV-CNGRC-EGFP vs. LV-EGFP; STDEV, standard deviation; LV, lentivirus; EGFP, enhanced green fluorescent protein. ${ }^{a}$ LV-CNGRC-3Flag -EGFP vs. LV; $\mathrm{P}<0.05$. ${ }^{\mathrm{L}} \mathrm{LV}-\mathrm{CNGRC}-3$ Flag -EGFP vs. LV-EGFP; $<<0.05$.
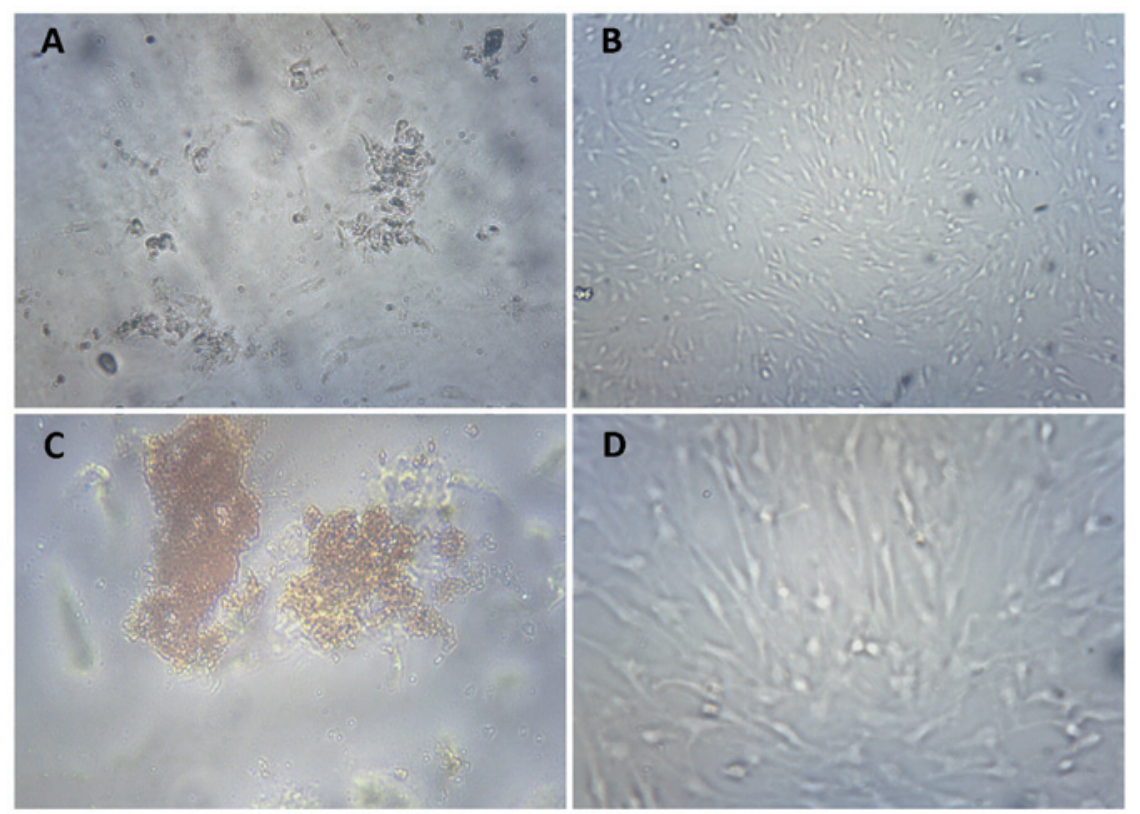

Figure 4. ADSCs following osteogenic induction. (A and B) ADSCs were cultured in induction medium (A, magnification, $\mathrm{x} 400)$ or normal medium (B, magnification, $\mathrm{x} 400)$ for 3 weeks. (C and D) Alizarin red stained calcium nodules in ADSCs cultured in induction medium (C, magnification, x2,000) but not in cells cultured in normal medium (D, magnification, x600). ADSCs, adipose stem cells.

A

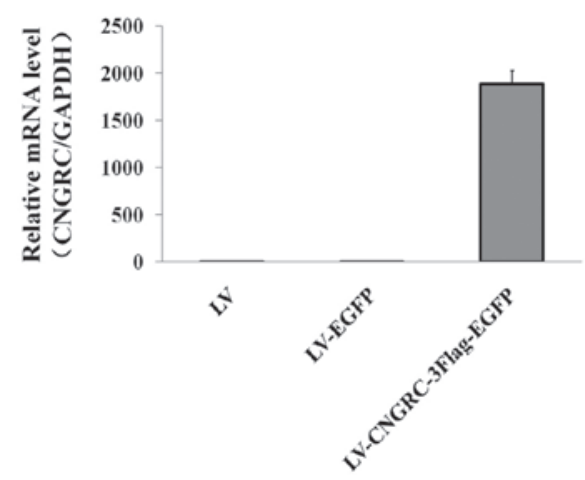

B

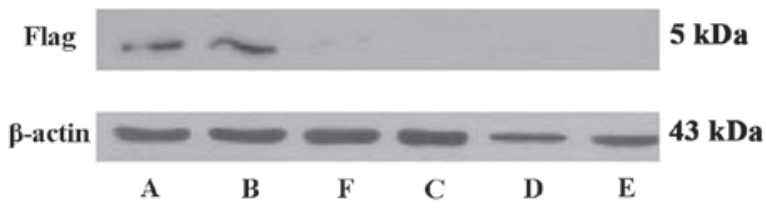

Figure 5. (A) CNGRC mRNA levels in ADSCs following transduction. Compared with non-transfected cells and cells transfected with LV-EGFP, cells transfected with LV-CNGRC-3Flag-EGFP contained significantly higher quantities of CNGRC mRNA. (B) Immunoblot showing expression of CNGRC peptide in (A, B and F) LV-CNGRC-3Flag-EGFP-transfected ADSCs, but not in (C and D) LV-EGFP-transfected ADSCs and (E) non-transfected cells. ADSCs, adipose stem cells.

Expression of CNGRC in $293 T$ cells and successful packaging of lentiviral vectors. Lentiviral vectors LV-EGFP and LV-CNGRC-3Flag-EGFP were co-transfected with Helper 1.0 and Helper 2.0 into 293T cells. CNGRC RNA levels in transfected and non-transfected cells were measured $72 \mathrm{~h}$ later by quantitative fluorescence PCR. CNGRC RNA levels in transfected cells were significantly higher $(\mathrm{P}<0.05)$ than levels in non-transfected and LV-EGFP-transfected cells (Table I and Fig. 5A), indicating successful expression of CNGRC in transfected cells. The titer of the lentivirus was calculated as $2.00 \mathrm{E}+8 \mathrm{TU} / \mathrm{ml}$, based on the difference in $\mathrm{Ct}$ values between non-transfected cells and transfected cells. 

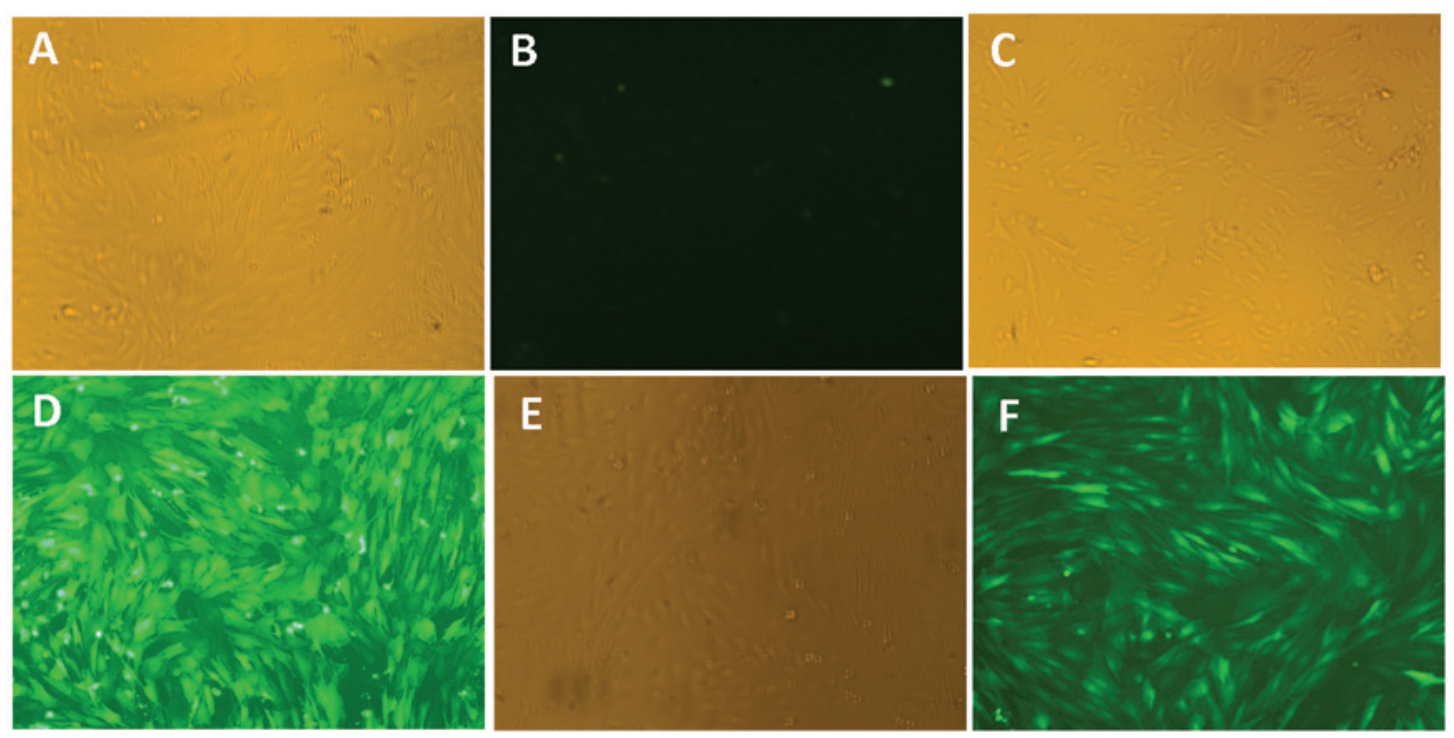

Figure 6. Expression of exogenous DNA in ADSCs following lentivirus infection. (A and B) Non-transfected ADSCs were observed under a (A) bright field and (B) fluorescence microscope. (C and D) LV-EGFP-transfected ADSCs were observed under a (C) bright field and (D) fluorescence microscope. (E and F) LV-CNGRC-3Flag-EGFP-transfected ADSCs were observed under a (E) bright field and (F) fluorescence microscope. Cells transfected with LV-EGFP and LV-CNGRC-3Flag-EGFP expressed high levels of EGFP, indicating successful transduction of lentivirus in cultured ADSCs. ADSCs, adipose stem cells.

Expression of exogenous DNA in ADSCs following lentivirus infection. To examine expression of exogenous DNA, transfected ADSCs were observed under a fluorescence microscope to detect the expression of EGFP after $72 \mathrm{~h}$ of transduction. LV-EGFP and LV-CNGRC-3Flag-EGFP demonstrated transduction efficiencies $>80 \%$. As shown in Fig. 6, EGFP was highly expressed in ADSCs transfected with the lentivirus. By contrast, no EGFP signal was detected in non-transfected cells. These results indicated that the exogenous gene could be expressed using lentiviral transduction.

Expression of CNGRC peptide in ADSCs following lentiviral transduction. To confirm expression of the CNGRC peptide, total proteins were extracted from ADSCs transfected with LV-EGFP, LV-CNGRC-3Flag-EGFP and without transduction, respectively. CNGRC-3Flag-EGFP was detected at the expected size by western blot analysis using anti-flag antibody in LV-CNGRC-3Flag-EGFP transfected cells, but was not detected in either non-transfected or LV-EGFP-transfected cells (Fig. 5B). The results clearly demonstrated expression of CNGRC peptide in ADSCs at the third passage, 12 days after infection with the lentivirus.

\section{Discussion}

ADSCs and bone marrow mesenchymal stem cells have similar phenotypes and differentiation potentials. However, it is easier to obtain large numbers of ADSCs than large numbers of mesenchymal stem cells (27). Bacigalupo et al (28) could obtain only $40 \mathrm{ml}$ of human bone marrow under local anesthesia, which yielded $\sim 1.2 \times 10^{5}$ stem cells. To obtain larger quantities of bone marrow mesenchymal stem cells, surgery must be performed under whole-body anesthesia, which may cause other complications. By contrast, Aust et al (29) revealed that up to $4 \times 10^{6}$ stem cells could be obtained from $200 \mathrm{ml}$ of adipose tissue, which was $\sim 30$-fold greater than the number of mesenchymal stem cells obtained from bone marrow. In addition, previous studies have reported successful transduction of ADSCs using a lentivirus and identified that transduction efficiency was significantly greater when using ADSCs rather than bone marrow mesenchymal stem cells (30-32). In the present study, it was demonstrated that ADSCs could be easily isolated from rat adipose tissue and that transduction efficiency using lentiviral vectors was $>80 \%$. Additionally, DNA expressing CNGRC peptide was successfully transfected using lentiviral vectors.

Microvessels surrounding cancer stem cells (stem cell niche) are important in regulating cancer stem cell numbers and their functions $(1,2)$. As mentioned previously, targeting of microvessels in stem cell niches has been suggested as an efficient method for treating tumors. NGR is a tri-peptide motif that can specifically bind to vascular endothelial cells of newly generated vessels in tumors (9). Based on this biological function, NGR-conjugates have been used in tumor diagnosis and treatment (12-17). However, the efficiency achieved when using this approach has been low due to the lack of a targeting vehicle. It was suggested that the efficient homing capacity of ADSCs could be exploited by using them as targeting vehicles to deliver NGR-conjugates specifically to cancer stem cell niches (3). To achieve this goal, it was first necessary to establish an ADSC cell line that stably expresses NGR-conjugates. In the present study, ADSC cell lines expressing the CNGRC peptide were established using lentiviral transduction. The mechanism by which these CNGRC-expressing ADSC cell lines migrate to cancer stem cell niches and eliminate newly generated microvessels remains to be elucidated. Future studies are to be conducted to assess the homing capacity of these ADSC cell lines and the in vivo vascular tropism effects of the CNGRC peptide. 


\section{Acknowledgements}

This study was supported by a grant from the National Natural Science Foundation of China (grant no. 81201680). The authors would like to thank Medjaden Bioscience Limited for assisting in the preparation of this manuscript.

\section{References}

1. Matsumoto Y, Iwasaki $\mathrm{H}$ and Suda T: Maintenance of adult stem cells: Role of the stem cell niche. Adult Stem Cells Springer Science+Business Media, LLC, pp35-55, 2011.

2. Takeda N, Jain R, LeBoeuf MR, et al: Interconversion between intestinal stem cell populations in distinct niches. Science 334: 1420-1424, 2011.

3. Kuhbier JW, Weyand B, Radtke C, et al: Isolation, characterization, differentiation and application of adipose-derived stem cells. Adv Biochem Eng Biotechnol 123: 55-105, 2010.

4. Josiah DT, Zhu D, Dreher F, et al: Adipose-derived stem cells as therapeutic delivery vehicles of an oncolytic virus for glioblastoma. Mol Ther 18: 377-385, 2010

5. Schäffler A and Büchler C: Concise review: adipose tissue-derived stromal cells-Basic and clinical implications for novel cell-based therapies. Stem Cells 25: 818-827, 2007.

6. Cho JA, Park H, Lim EH, et al: Exosomes from ovarian cancer cells induce adipose tissue-derived mesenchymal stem cells to acquire the physical and functional characteristics of tumor-supporting myofibroblasts. Gynecol Oncol 123: 379-386, 2011.

7. Rowan BG, Gimble JM, Sheng M, et al: Human adipose tissue-derived stromal/stem cells promote migration and early metastasis of triple negative breast cancer xenografts. PLoS One 28: e89595, 2014.

8. Park YM, Yoo SH, Kim SH: Adipose-derived stem cells induced EMT-like changes in H358 lung cancer cells. Anticancer Res 33: 4421-4430, 2013.

9. Hou L, Zhao X, Wang P, et al: Antitumor activity of antimicrobial peptides containing CisoDGRC in CD13 negative breast cancer cells. PLoS One 8: e53491, 2013.

10. Robak L A, Venkatesh K, Lee H, et al: Molecular basis of the interactions of the Nogo-66 receptor and its homolog NgR2 with myelin-associated glycoprotein: development of NgROMNI-Fc, a novel antagonist of CNS myelin inhibition. J Neurosci 29: 5768-5783, 2009.

11. Curnis F, Cattaneo A, Longhi R, et al: Critical role of flanking residues in NGR-to-isoDGR transition and CD13/integrin receptor switching. J Biol Chem 285: 9114-9123, 2010.

12. Ndinguri MW, Solipuram R, Gambrell RP, et al: Peptide targeting of platinum anti-cancer drugs. Bioconjug Chem 20: $1869-1878,2009$

13. Yokoyama Y and Ramakrishnan S: Addition of an aminopeptidase $\mathrm{N}$-binding sequence to human endostatin improves inhibition of ovarian carcinoma growth. Cancer 104: 321-331, 2005.
14. Liu Y, Wei H, Zhen Y, et al: Relationship between receptor expression on cell surface of several solid tumors and tumor sensitivity to IFN- $\alpha$ 2a. Journal of the Fourth Military Medical University 10: 865-868, 2008 (In Chinese).

15. Meng J, Ma N, Yan Z, et al: NGR enhanced the anti-angiogenic activity of tum-5. J Biochem 140: 299-304, 2006.

16. Corti A, Pastorino F, Curnis F, et al: Targeted drug delivery and penetration into solid tumors. Med Res Rev 32: 1078-1091, 2012.

17. Curnis F, Sacchi A and Corti A: Improving chemotherapeutic drug penetration in tumors by vascular targeting and barrier alteration. J Clin Invest 110: 475-482, 2002.

18. Podolska K, Stachurska A, Hajdukiewicz K, et al: Gene therapy prospects - intranasal delivery of therapeutic genes. Adv Clin Exp Med 21: 525-534, 2012.

19. Christ M, Lusky M, Stoeckel F, et al: Gene therapy with recombinant adenovirus vectors: evaluation of the host immune response. Immunol Lett 57: 19-25, 1997.

20. Zhou YF: In vitro study of HCN4 transfected biological pacemaker cells using lentivirus. Soochow University, pp47-49, 2009.

21. Zhao L, Wu J, Zhou H, et al: Local gene delivery for cancer therapy. Curr Gene Ther 11: 423-432, 2011.

22. Liu YP and Berkhout B: miRNA cassettes in viral vectors: problems and solutions. Biochim Biophys Acta 1809: 732-745, 2011.

23. Nery AA, Nascimento IC, Glaser T, et al: Human mesenchymal stem cells: from immunophenotyping by flow cytometry to clinical applications. Cytometry A 83: 48-61, 2013.

24. Zuk PA, Zhu M, Ashjian PH, et al: Human adipose tissue is a source of multipotent stem cells. Mol Biol Cell 13: 4279-4295, 2002.

25. Rangappa S, Fen C, Lee E H, et al: Transformation of adult mesenchymal stem cells isolated from the fatty tissue into cardiomyocytes. The Annals of thoracic surgery 75: 775-779, 2003.

26. Zuk PA, Zhu M, Mizuno H, et al: Multilineage cells from human adipose tissue: Implications for cell-based therapies. Tissue Eng 7: 211-228, 2001.

27. Araña M, Mazo M, Aranda P, et al: Adipose tissue-derived mesenchymal stem cells: isolation, expansion and characterization. Methods Mol Biol 1036: 47-61,2013.

28. Bacigalupo A, Socié G, Schrezenmeier H, et al: Bone marrow versus peripheral blood as the stem cell source for sibling transplants in acquired aplastic anemia: survival advantage for bone marrow in all age groups. Haematologica 97: 1142-1148, 2012.

29. Aust L, Devlin B, Foster SJ, et al: Yield of human adipose-derived adult stem cells from liposuction aspirates. Cytotherapy 6: 7-14, 2004.

30. Dragoo JL, Choi JY, Lieberman JR, et al: Bone induction by BMP-2 transduced stem cells derived from human fat. J Orthop Res 21: 622-629, 2003.

31. Sun XZ, Liu GH, Wang ZQ, et al: Over-expression of VEGF165 in the adipose tissue-derived stem cells via the lentiviral vector. Chin Med J (Engl) 124: 3093-3097, 2011.

32. Liu Y, Chen C, He H, et al: Lentiviral-mediated gene transfer into human adipose-derived stem cells: role of NELL1 versus BMP2 in osteogenesis and adipogenesis in vitro. Acta Biochim Biophys Sin (Shanghai) 44: 856-865, 2012. 